\title{
An Efficient Signature Computation Method
}

Maroware overhead, impact on system performance, and fault coverage are key variables a designer must consider in deciding to use BIST (built-in self-test) in a design. Once the designer completes a design with BIST capability, its hardware overhead and system performance become known deterministically. But the designer must rely on probabilistic results about the aliasing aspect of fault coverage. (A vast body of literature deals with the aliasing problem.) For high fault coverage, that approach is unsatisfactory. Indeed, the only way to obtain a deterministic answer about fault coverage is to simulate the circuit and the BIST environment for each fault in the circuit. While simulating BIST circuits, designers often use pseudorandom pattern generators and compactors such as linear-feedback shift registers (LFSRs). The simulation is costly because it entails the simulation of the generator, the faulty circuit, and the compactor for large numbers of test vectors, without fault dropping.

Advances in fault simulation techniques have reduced simulation time

An earlier version of this article was presented at the Fifh Intemational Conference on VLSI Design, Bangalore, India, January 1992.

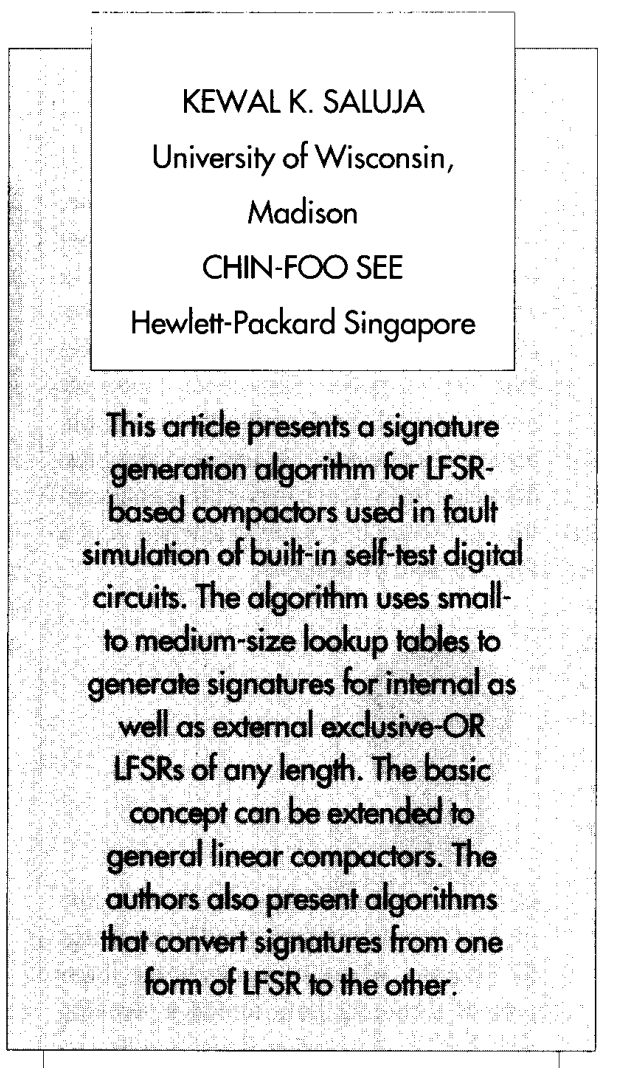

considerably, but research has done little to improve the efficiency of compaction, or signature computation, algorithms. ${ }^{1-3}$ Recently, Lambidonis, Ivanov, and Agarwal $^{1}$ proposed a table-driven compaction algorithm applicable to single-input linear compactors, which is similar to the algorithm presented in this article.
We propose a signature computation algorithm based on methods proposed earlier. ${ }^{2,45}$ We restrict our discussion and application of the algorithm to single-input LFSRs, although the basic concepts described here can be applied to arbitrary linear compactors with multiple inputs.

\section{Preliminaries and notation}

Figure 1 shows a popular realization of an LFSR. It is an internalexclusive-OR (internalXOR) implementation and a true serial divisor. In the figure each $g_{i}$ is either a 1 (closed connection) or a 0 (open connection). We express the LFSR as a polynomial, $g(x)$, which characterizes the connections. Similarly, we express the input stream as a polynomial, $I(x)$, and the contents of the LFSR as a polynomial, $R_{f}(x)$. We perform division by taking $I(x)$ as dividend and $g(x)$ as divisor, with $R_{l}(x)$ the remainder. After we fully process $I(x)$, the final value of $R_{R}(x)$, called the internal signature, is the check bits for a cyclic redundancy check (CRC) code. ${ }^{6}$

Figure 2 shows an altemative LFSR realization, an external-XOR implementation. We use $I(x)$ to express the input stream, $g(x)$ to express the LFSR with external-XOR, and $R_{E}(x)$ to express the 
contents of the LFSR. We call the final $R_{E}(x)$ the external signature.

We now explain how to construct lookup tables and use them to compute the intemal signature. Consider the situation shown in Figure 3a. Let us assume that the contents of the LFSR are $S^{*}$, and an 8-bit input to the LFSR is $i^{*}$. We wish to compute the contents of the LFSR after $i^{*}$ has been shifted into the LFSR. Using the principle of superposition (linearity), we partition the problem into two parts, as shown in Figure 3b. Clearly, for an internal-XOR LFSR of length 8 or more bits, with an intemal state of 0 and an input $i^{*}$, the state of the LFSR after eight shifts will be $i^{*}$, as shown in Figure $3 \mathrm{c}$. On the other hand, the state $S^{* *}$ shown in Figure $3 \mathrm{c}$ is the state of the LFSR after we start from state $S^{*}$ and shift eight zeros into it. We must determine this state.

We can obtain $S^{\star *}$ from $S^{\star}$, using precomputed lookup tables. To explain the process, we assume that the LFSR is 16 bits long and we use the pictorial representation developed in Figure 3. Figure 4 shows the complete process. In Figure 4a, $S_{1}{ }^{*}$ and $S_{2}{ }^{*}$ are the two 8-bit components of $S^{*}$, and we assume the input to the LFSR is 0. Again using the principle of superposition in linear systems, we partition the problem into two parts (Figure $4 \mathrm{~b}$ ). Figure 4c shows the two-part state of the intemal-XOR LFSR after eight shifts. It is now evident that we need to compute $S$, the state of the LFSR after eight shifts, provided the initial content of the LFSR is $S_{1}{ }^{*}$

Knowing the polynomial or the feedback structure of the LFSR, we can construct a table off line for every possible value of $S_{1}{ }^{*}$. If $S_{1}{ }^{*}$ is 8 bits, the table will have $2^{8}=256$ entries, and each entry for our example will be 16 bits wide. Finally, by XORing $S, S_{2}{ }^{*}$, and $i^{*}$, we compute the state $S^{* *}$. Thus, the process of finding $S^{* *}$ from $S^{*}$ and $i^{*}$ involves one table lookup and two XOR operations. Further, if we look carefully at Figure $3 \mathrm{c}$ and Figure $4 \mathrm{c}$, we see that $S_{2}{ }^{*}$ and $i^{*}$ need not be XORed. Instead, they can be concatenated, a step we can integrate into the

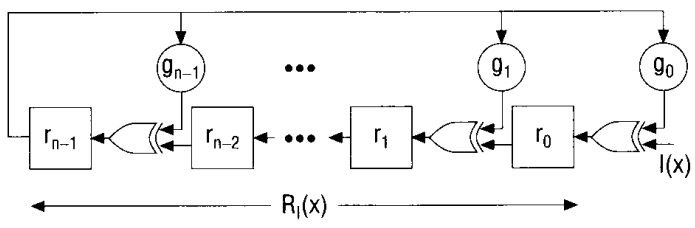

Figure 1. Internal-XOR LFSR

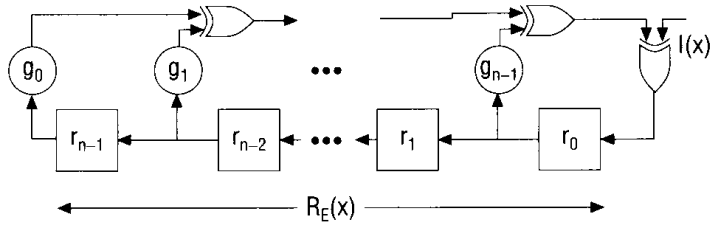

Figure 2. External-XOR LFSR.

formation of the table. ${ }^{5}$

Before proceeding to the next section, we point out that for a given $I(x)$ and $g(x)$, the values of $R_{f}(x)$ and $R_{E}(x)$ are different, but there is a one-to-one mapping between internal and external signatures. ${ }^{6}$ Later in the article we present a technique to convert an internal signature to an external signature and vice versa. The conversion technique takes an amount of time proportional to the LFSR's length and independent of the input data stream's length. Moreover, our scheme works for any polynomial (not just primitive polynomials) characterizing the LFSR.

\section{Signature computation}

Our signature computation algorithm is based on the explanation given in the preceding section. (Sarwate presents an alternative explanation. ${ }^{5}$ ) Figure 5 (page 24)presents a pictorial representation of the algorithm; each $A(i)$ is a byte-long input to the LFSR, and the input stream consists of $m$ bytes.

The algorithm consists of two parts. The first part constructs the lookup tables by simulating the LFSR or by computing for all possible values of $S_{1}{ }^{*}$ as explained earlier. Next, the algorithm processes the input stream and computes the check bits, using the lookup (a)
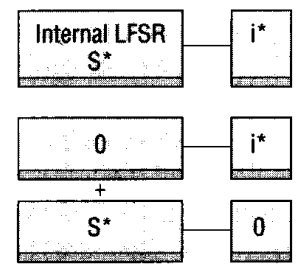

(b)

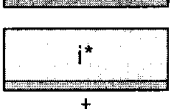

(c)

$\mathrm{S}^{\star *}$

Figure 3. Processing an input stream by an internal-XOR LFSR: internal state (a); partitions (b); states after processing (c).
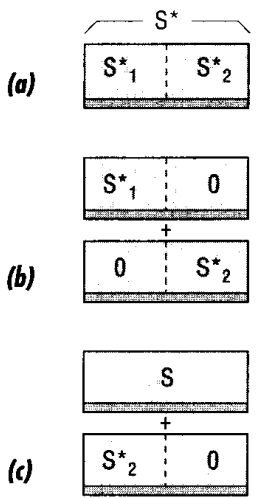

Figure 4. Determining the state of an internal-XOR LFSR: initial state (a); partition state (b); state after eight shifts (c). 


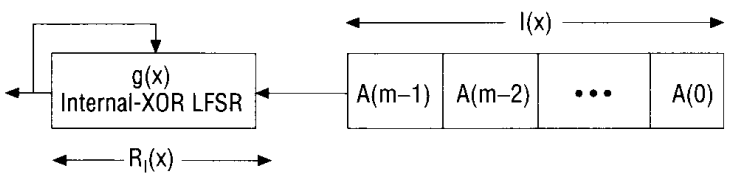

Figure 5. General representation of signature computation algorithm.

tables. It is this part of the algorithm that is of interest to us in this section. The algorithm variations for computing $8-16$, 24 , and 32-bit signatures are shown in the box on page 25 . They are in pseudo $C$ language. In each case, the size of the lookup tables is $2^{8}$ because each $A(i)$ is 1 byte ( 8 bits) long.

Algorithm 1 first initializes the 8-bit register $\mathrm{C} 0$ to zero. Then, it takes $m$ cycles to process $m$ bytes of input. The byte $A(m-1)$, the most significant byte, is processed first. $T$, a temporary variable, is used as an index into the lookup table f0. When all the input bytes have been processed, the remainder (the check bits or the signature) is left in $\mathrm{C} 0$.

In the algorithms for computing 16-, 24 , and 32-bit signatures, we use the variables $\mathrm{C} 0, \mathrm{C} 1, \mathrm{C} 2$, and $\mathrm{C} 3$ to form the signature. Each of these Civariables is 8 bits long. Similarly, f0, $\mathrm{fl}, \ldots$, represent lookup tables. (We give a method of extending these algorithms to nonmultiples of 8-bit signatures in the earlier version of this article. ${ }^{2}$ )

Algorithm 2 is Sarwate's algorithm for computation of 16 -bit signatures. ${ }^{5}$ The algorithm produces the signature for an input stream by appending a stream of zeros. Furthermore, it modifies the input stream while generating the signature. To produce the correct signature for a given input stream, we must modify the algorithm or follow it with some postprocessing. Algorithm 3 is a modified version that produces the correct signature and does not modify the input stream.

Algorithms 4 and 5, for 24- and 32-bit LFSRs, are similar to the 8-bit and 16-bit algorithms, except that they require more lookup tables and more operations per cycle.

\section{Signature conversion}

If internal-XOR and extemal-XOR LFSRs are characterized by the same polynomial, $g(x)$, a one-to-one relationship exists between $R_{f}(x)$ and $R_{E}(x){ }^{6}$ That means that conversion from one form of signature to the other is always possible and the result is always unique. Conversion may be required for any of several reasons:

- If we know either the internal or the external signature, we can obtain the other form of signature by conversion, instead of reprocessing all the input stream to derive the other form. One of the most time-consuming steps in reprocessing the input stream is reading the input file, which we avoid in the conversion process.

- We may wish to initiate signature generation as soon as we choose the
LFSR polynomial but before we decide on the actual implementation of the LFSR (intemal or extemal).

- Sometimes we need to know both signatures before choosing one form over the other-for example, when the storage or hardware realization of one signature is superior to the other in the BIST environment. In such a case we can simulate either the internal or the extemal signature and then perform the conversion. This reduces the processing time to approximately half the time of simulating both forms of LFSRs.

- Simulating one form may be faster than simulating the other form. In that case we can compute the signature with the faster form, and convert it to the other form.

In the following explanation of the conversion processes, we have not included formal proofs as they are unduly long, but they are easy to deduce by logical reasoning.

Internal to external. If we define $I(x), g(x)$, and $R_{l}(x)$ as in Figure 1 , where $I(x)$ is the input polynomial, $g(x)$ is the division polynomial, and $R_{f}(x)$ is the internal-XOR LFSR signature, then

$$
I(x)=Q(x) \cdot g(x)+R_{f}(x)
$$

where $Q(x)$ is the quotient polynomial. Similarly, if we process the same input through the external-XOR LFSR with the same $I(x)$ and $g(x)$, and $R_{E}(x)$ is the extemal-XOR LFSR signature, there also exists a relation

$$
I(x)=Q(x) \cdot g(x)+f\left(R_{E}(x)\right)
$$

where $R_{f}(x)=f\left(R_{E}(X)\right)$, and the function $f$ is one-to-one. Note that the quotient polynomial is the same for both forms, but the signatures $\left(R_{f}(x)\right.$ and $\left.R_{E}(x)\right)$ may be different.

To convert $R_{f}(x)$ to $R_{E}(x)$, we first ini-

Figure 6. External-XOR LFSR with $g(x)=x^{4}+x^{2}+x+1$. 
tialize the external-XOR LFSR to an allzero state. Then we input $R_{r}(x)$ into the external-XOR LFSR. After $n$ cycles (where $n$ is the length of the signature), the external signature, $R_{E}(x)$, remains in the LFSR.

We demonstrate this process through an example. Let us consider $g(x)=x^{4}+$ $x^{2}+x+1$ (a nonprimitive polynomial). Figure 6 gives the extemal-XOR LFSR for this polynomial. For $R_{f}(x)=1101$, we can verify that $R_{E}(x)$ is 1111 . This is a unique value of $R_{E}(x)$ for $R_{f}(x)=1101$. Table 1 shows all possible 4-bit signatures $R_{f}(x)$ and $R_{E}(x)$ derived through this procedure and the mapping between them for the polynomial $g(x)$ in this example.

External to internal. In the preceding subsection, we demonstrated that with $R_{f}(x)$ as input to the external-XOR LFSR, we can obtain $R_{E}(x)$ in the LFSR. To derive the internal signature, $R_{f}(x)$, from the external signature, $R_{E}(x)$, we load the extemal-XOR LFSR with $R_{E}(x)$, and compute "backward" till the contents of the register become zero. At this stage the value of $R_{f}(x)$ appears as $I(x)$.

Table 1. Signatures of $g(x)=x^{4}+x^{2}+x+1$

\begin{tabular}{|cc|}
$\begin{array}{c}\text { Internal signature } \\
\boldsymbol{r}_{3} \boldsymbol{r}_{\mathbf{2}} \boldsymbol{r}_{\mathbf{1}} \boldsymbol{r}_{\mathbf{0}}\end{array}$ & $\begin{array}{c}\text { External signature } \\
\boldsymbol{r}_{\mathbf{3}} \boldsymbol{r}_{\mathbf{2}} \boldsymbol{r}_{\mathbf{1}} \boldsymbol{r}_{\mathbf{0}}\end{array}$ \\
\hline 00000 & 0000 \\
0001 & 0001 \\
0010 & 0010 \\
0011 & 0011 \\
01100 & 0101 \\
01101 & 0100 \\
01110 & 0111 \\
0111 & 0110 \\
1000 & 1011 \\
1001 & 1010 \\
1010 & 1001 \\
1011 & 1000 \\
1100 & 1110 \\
1101 & 1111 \\
1110 & 1100 \\
1111 & 1101 \\
\hline
\end{tabular}

\section{Signature computation algorithms}

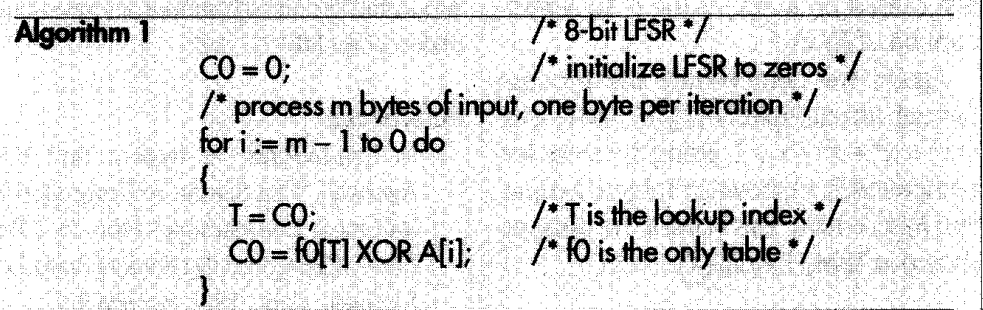

Agoritim 2

* 16-bit LFSR *

$\mathrm{CO}=\mathrm{Cl}=0 ;$
$l^{*}$ process $\mathrm{m}$ bytes of inpult, one byte per iteration $\%$

for $i=m-1$ to 0 do

I

$\mathrm{T}=\mathrm{CI}$ XOR Ali]; $\quad I^{*} \mathrm{~T}$ is the lookup index $\%$

$\mathrm{Cl}=\mathrm{CO}$ XOR fi[T]; $1 *$ process bit $15-8$ of LFSR $\%$

1

7 * process bit 7-0 of LFSR *

Agorithm 3

/* Two tables, $\mathrm{fO}$ and $\mathrm{fl}$, needed */

$\mathrm{CO}=\mathrm{Cl}=0$;

$/$ initialize LFSR to zeros $\%$

for $i:=m-1$ to 0 do

I

$\mathrm{T}=\mathrm{Cl}$

${ }^{*} \mathrm{~T}$ is the index of tables *

$\mathrm{Cl}=\mathrm{COXOR} \mathrm{f}[1 \mathrm{~T}] ; \quad / *$ process upper 8 bits of LFSR $*$ ]

)

$\mathrm{CO}=\mathrm{FO}[\mathrm{T}] \times \mathrm{OR} \mathrm{A}[\mathrm{i}] ; \quad 1^{*}$ process lower 8 bits of LFSR $\%$

Agoritim 4

/ Three tables, $10, \mathrm{f1}$, and $\$ 2$, needed *

/24-bit LFSR * +

$\mathrm{CO}=\mathrm{Cl}=\mathrm{C} 2=0$; $\quad{ }^{*}$ initialize LFSR to zeros *I

for $i:=m-1$ to 0 do

I

$\mathrm{T}=\mathrm{C} 2 ; \quad \mathrm{I}, \mathrm{T}$ is the index of tables $\%$

$\mathrm{C} 2=\mathrm{C} 1$ XOR $\{2 \mathrm{~T}] ; \quad 1 \%$ process bit $23-16$ of LFSR $\%$

$\mathrm{Cl}=\mathrm{CO}$ XOR fIIT]; $\quad /$ process bit $15-8$ of LFSR $\%$

1)

$\mathrm{CO}=$ [O[T] XOR A[i]; $/ *$ process bit $7-0$ of LFSR *

Agoritim 5

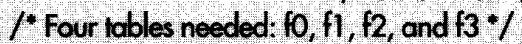

$\mathrm{CO}=\mathrm{Cl}=\mathrm{C} 2=\mathrm{C} 3=0$; $\%$ initialize IFSR to zeros *

for $i:=m-1$ to 0 do

I

$\mathrm{T}=\mathrm{C} 3$;

= 2 XOR F[T]. $\quad 7^{*}$ process bit $31-24$ of IFSR *

$\mathrm{C} 2=\mathrm{C} 1$ XOR 2 [T]; $\quad I *$ process bit 23-16 of LFSR *

$\mathrm{Cl}=\mathrm{CO}$ XOR f1[T]; $\quad / *$ process bit $15-8$ of LFSR * 
Note that during the backward computation, $Q(x)$ must be set to zero. Also, the first output bit at $I(x)$ is the least significant bit of $R_{f}(x)$.

Again, we demonstrate the process through an example. Let $R_{E}(x)=1011$ and $g(x)=x^{4}+x^{2}+x+1$. Using the steps for computing $R_{f}(x)$ shown in Table 2 , we find that $R_{f}(x)=1000$. This value is the same as shown in Table 1 for $R_{E}(x)=1011$.

THE Alcormum We have presented computes signatures very efficiently. It requires $k n /\left[\log _{2} \text { (table_size) }\right]^{2}$ operations to compute the signatures, where $k$ and $n$ are the number of bits in the input stream and in the signature register respectively, and table_size is the size of the lookup table. The algorithm in all its variations is written for table_size $=256$. Thus, it has small to moderate memory requirements and is faster than similar algorithms reported in the literature. ${ }^{1,3}$ Using the signature generation and conversion algorithms, designers can determine signatures for both internal-XOR and extemal-XOR LFSRs by fast software computation. एक्षि

\section{Acknowledgment}

The work described in this article was supported in part by National Science Foundation grant MIP-9111886.

\section{References}

1. D. Lambidonis, A. Ivanov, and V.K. Agarwal, "Fast Signature Computation for Linear Compactors," Proc. Int'l Test Conf., IEEE Computer Society Press, Los Alamitos, Calif., 1991, pp. 808-817.

2. C.F. See and K.K. Saluja, "An Efficient Method for Computation of Signatures," Proc. Fifth Int'l Conf. VLSI Design, IEEE CS Press, 1992, pp. 245-250.

3. S.B. Tan et al., "A Fast Signature Simulation Tool for Built-In Self-Testing Circuits," Proc. 24th Design Automation Conf., IEEE CS Press, 1987, pp. 17-25.

4. G. Griffiths and G.C. Stones, "The TeaLeaf Reader Algorithm: An Efficient Implementation of CRC-16 and CRC-32," Comm. ACM, Vol. 30, No. 7, July 1987, pp. $617-620$.

5. D.V. Sarwate, "Computation of Cyclic Redundancy Checks via Table Lookup," Comm. ACM, Vol. 31, No. 8, Aug. 1988, pp. 1008-1013.

6. W.W. Peterson and E.J. Weldon, Jr., Error-Correcting Codes, 2nd ed., MIT Press, Cambridge, Mass., 1972.

Table 2. Example computation of internal signature from external signature.

\begin{tabular}{|rrrrrrl|}
\hline $\mathbf{Q}(\mathbf{x})$ & $\mathbf{r}_{\mathbf{3}}$ & $\mathbf{r}_{\mathbf{2}}$ & $\mathbf{r}_{\mathbf{1}}$ & $\mathbf{r}_{\mathbf{0}}$ & $\mathbf{l}(\mathbf{x})$ & \\
\hline 0000 & 1 & 0 & 1 & 1 & - & $/^{*}$ set 1011 in register ${ }^{*} /$ \\
000 & 0 & 1 & 0 & 1 & 0 & $/^{*}$ process for four clock cycles ${ }^{*} /$ \\
00 & 0 & 0 & 1 & 0 & 00 & \\
0 & 0 & 0 & 0 & 1 & 000 & \\
- & 0 & 0 & 0 & 0 & 1000 & \\
\hline
\end{tabular}

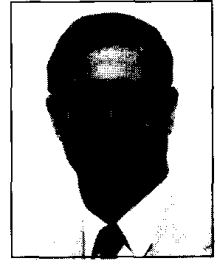

Kewal K. Saluja is a professor in the Department of Electrical and Computer Engineering at the University of Wisconsin-Madison, where he teaches logic design, computer architecture, microprocessor-based systems, and VLSI design and testing. Previously, he worked at the University of Newcastle, Australia. He has also held visiting and consulting positions at the University of Southem Califomia, the University of lowa, and Hiroshima University. His research interests include design for testability, fault-tolerant computing, VLSI design, and computer architecture. He is an associate editor of the Joumal of Electronic Testing: Theory and Applications. Saluja received the BE from the University of Roorkee, India, and the MS and the $\mathrm{PhD}$ in electrical and computer engineering from the University of lowa.

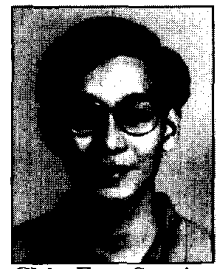

Chin-Foo See is a production engineer at Hewlett-Packard Singapore. He is responsible for the HP 75LX Palmtop Computer production, quality assurance, testing, line automation, and cost reduction programs. He received the BSEE from the University of Southwestem Louisiana, Lafayette, and the MSEE in electrical and computer engineening from the University of Wisconsin-Madison, where he participated in the research described in this article. His areas of interest include VLSI design, testing, and computer architecture.

Address correspondence about this article to Kewal K. Saluja, Dept. of Electrical and Computer Engineering, University of Wisconsin, Madison, WI 53706; e-mail: saluja@engr.wisc.edu.

IEEE DESICN \& TEST OF COMPUTERS 